\title{
Computed Tomography Study File
}

National Cancer Institute

\section{Source}

National Cancer Institute. Computed Tomography Study File. NCI Thesaurus. Code

C115499.

A collection of computed tomography files pertaining to a clinical study. 